ARTIGO

\title{
A Covid-19 e a volta às aulas: ouvindo as evidências*
}

\author{
João Batista Araujo e Oliveira ${ }^{\text {a }}$ \\ Matheus Gomes b \\ Thais Barcellos ${ }^{c}$
}

\section{Resumo}

Paralisadas pelo pânico decorrente da pandemia provocada pela Covid-19, o fechamento das escolas trará consequências para a aprendizagem dos alunos. Este artigo examina situações de paralisação, uso do tempo e impacto de tecnologias no desempenho escolar. O exame das evidências sugere como pouco promissoras as estratégias de aumento intensivo de tempo ou recurso a tecnologias. Caminhos mais promissores estariam associados a diagnósticos, intervenções estruturadas mais adequadas ao perfil dos professores, melhor aproveitamento do tempo, uso judicioso dos deveres de casa, redução do absenteísmo e tutoria intensiva de alta qualidade focada nos alunos de maior risco.

Palavras-chave: Covid-19. Interrupção do Calendário Escolar. Educação Baseada em Evidências.

\section{Introdução}

De acordo com a Organização das Nações Unidas para a Educação, a Ciência e a Cultura (UNESCO, 2020), no início de maio de 2020, 186 países ou regiões fecharam escolas, total ou parcialmente, para conter a disseminação da Covid-19, atingindo cerca de $70 \%$ dos alunos. Esse fechamento afetou o calendário escolar, sendo incerto o seu impacto sobre o aprendizado dos alunos. Diferenças no rigor da quarentena, na sua duração e nas estratégias adotadas pelas famílias e escolas são apenas alguns dos fatores que poderão influenciar a trajetória desses alunos.

\footnotetext{
* Agradecemos aos dois pareceristas anônimos, ao conselho editorial da revista, a Guilherme Hirata, Simon Schwartzman e Cláudio de Moura Castro por seus valiosos comentários e sugestões para este artigo. As opiniões aqui expressas são de inteira responsabilidade dos autores.

a Instituto Alfa e Beto, Brasília, DF, Brasil.

b IDados, Rio de Janeiro, RJ, Brasil.

c IDados, Rio de Janeiro, RJ, Brasil.
} 
Em meio à pandemia atual, a ciência, apoiada em evidências empíricas, passou a ganhar importância irrefutável. Assim, entender o impacto da Covid-19 sobre a Educação, baseando-se no que dizem as evidências científicas é relevante para sociedade. Nesse contexto, o presente artigo tem como objetivos investigar o impacto do fechamento das escolas sobre o desempenho dos alunos no curto prazo e suas trajetórias a longo prazo. Também objetiva refletir sobre as potenciais intervenções de curto prazo que poderiam mitigar suas consequências. Para isso, foi realizada uma análise da literatura, na qual foram selecionados trabalhos acadêmicos recentes em publicações com revisão de pares. Em alguns casos, devido à lacuna literária, ou tempo insuficiente para publicação, investigaramse artigos científicos ainda não publicados, sempre mantendo os critérios de rigor científico.

O presente estudo organiza-se em mais cinco partes, além desta introdução. A seção dois apresenta evidências a respeito do impacto do fechamento de escolas, por períodos mais ou menos longos. A seção três examina intervenções potencialmente óbvias ou promissoras, mas cujas eficácias não encontram respaldo nas evidências e geram custos injustificados. A seção quatro examina limitações estruturais que dificultam a implementação de medidas efetivas, ou potencialmente promissoras, - com ênfase nas limitações associadas à qualidade do professor e no acesso a tecnologias. A quinta seção apresenta evidências sobre o que poderia funcionar dentro das circunstâncias e dos recursos disponíveis no país. O estudo é concluído com reflexões sobre o papel das evidências, suas limitações diante dos formidáveis desafios apresentados pela interrupção do calendário escolar e as oportunidades factíveis.

\section{O fenômeno do "Summer learning loss"}

No decorrer da história, ainda que não na mesma magnitude que atualmente, diferentes eventos já alteraram o funcionamento regular das escolas. Em 1916, uma epidemia de poliomielite nos Estados Unidos resultou em uma quarentena e consequente fechamento das escolas nos primeiros dois meses do ano letivo. Isso resultou em evasão, tal que a coorte atingida pela quarentena alcançou uma escolaridade média menor ao longo da vida (MEYERS; THOMASSON, 2017). Em 1990, uma greve de professores em uma comunidade na Bélgica paralisou as escolas por quase seis meses. De modo semelhante ao caso da poliomielite, Belot e Webbink (2010) verificaram que a escolaridade média dos alunos afetados caiu em relação a de alunos não afetados pela greve. Mais recentemente, em 2005, os furacões Katrina e Rita levaram ao fechamento de escolas e à realocação de alunos entre escolas no sul dos Estados Unidos. Como resultado, houve queda no desempenho de Matemática (SACERDOTE, 2012). 
Os fenômenos descritos acima permitem estudar suas consequências, justamente, porque geram uma mudança súbita e não planejada, resultando em um experimento natural. Entretanto, essa análise é complicada devido à falta de dados, pois não se trata de eventos controlados. Contudo, escolas fecham anualmente de forma planejada para as férias escolares, o que cria uma oportunidade para estudar esse efeito sobre o aprendizado. Em tese, durante esse período de dois a três meses de paralisação, ocorreria uma desaceleração, ou perda de aprendizado, obtido durante o período letivo. Esse tema foi estudado por diversos autores em alguns países e se tornou conhecido como Summer Learning Loss ou Summer Slide. Obviamente, as férias planejadas para o final do ano escolar, com duração prédefinida, diferem do período de incerteza gerado pela pandemia da Covid-19.

A literatura reporta resultados ambíguos a respeito do impacto da parada nas férias. Estudos realizados com dados mais recentes, mas ainda não publicados em revistas científicas, mostraram um efeito negativo das férias de verão nos EUA de até 30\% em Linguagem e Matemática (ATTEBERRY; MCEACHIN, 2020; KUHFELD, 2019; KUHFELD; SOLAND, 2020). No entanto, outros estudos, nomeadamente de Benson e Borman (2010), Hippel, Workman e Downey (2018), Patton e Reschly (2013), Rambo-Hernandez e McCoach (2014) e Vale et al. (2013), apontaram para uma perda nula ou quase totalmente nula. Segundo Hippel e Hamrock (2019), a taxa de aprendizado durante o verão após o primeiro ano escolar seria próxima de zero (entre 0,05 e -0,02 desvio-padrão para cada mês de férias), mas sem prejuízos ao desempenho final do aluno.

Um outro modo de analisar o efeito das férias é estudando os casos em que a paralização foi encurtada para redistribuir os dias letivos ao longo do ano mais uniformemente. Essa literatura encontrou efeito positivo pequeno, ou nulo, sobre o desempenho do aluno. Isso corrobora a evidência de que as férias de verão não produzem resultados tão deletérios sobre os alunos (MCMULLEN; ROUSE, 2012; WILMORE; SLATE, 2012).

Outra questão recorrente na literatura é a desigualdade de desempenho entre grupos - por exemplo, negros e brancos, alto e baixo nível socioeconômico - durante as férias. Conforme Atteberry e McEachin (2020), há poucas evidências de que essas desigualdades se acumulem. Em geral, quanto ao nível socioeconômico, os resultados sugerem que não há um aumento na diferença de desempenho e, quando essa ocorre é sutil. Por sua vez, as diferenças no desempenho escolar entre raças podem até diminuir durante as férias (HIPPEL; HAMROCK, 2019; HIPPEL; WORKMAN; DOWNEY, 2018; QUINN, 2015; QUINN; LE, 2018). O que parece ocorrer é uma redução dos ganhos de conhecimento aferidos por testes, aplicados logo após longas paralizações. No entanto, essas diminuições 
tendem a desaparecer no curto prazo, com o retorno regular das aulas. Assim, as diferenças entre alunos de diferentes grupos socioeconômicos tendem a manterse constantes durante o processo escolar. Ou seja, ainda que possa haver perdas, essas não são permanentes.

Há um consenso de que a aprendizagem medida pelos testes de desempenho escolar utilizados nesses estudos não aumente durante as férias. Isso ocorre a despeito das atividades realizadas pelos alunos nesse período fora da escola. Em contrapartida, as evidências não refutam, nem confirmam a hipótese de perda de aprendizagem durante o verão e, nos estudos em que há perda, essa varia em termos de magnitude.

\section{Estratégias potencialmente promissoras}

Dada a evidência empírica a respeito do impacto de fechamento das escolas (planejados ou não) sobre a aprendizagem, permanecem incertos a ocorrência, a magnitude das perdas e os efeitos sobre diferentes grupos de alunos. Em face às incertezas, o mais intuitivo no retorno às aulas seria ampliar a jornada diária, de modo que a carga horária incorrida nos 200 dias letivos se encaixe nos dias letivos restantes pós-pandemia. Dessa forma, o currículo seria cumprido e não afetaria o ano letivo de 2021. Dependendo da duração da quarentena, isso poderia requerer um esforço equivalente a implementar o tempo integral em todas as escolas do país. Cabe examinar, portanto, a literatura que trata da duração do ano escolar e seu impacto no desempenho dos alunos.

Estudos como os de Long (2013), Lopez-Agudo e Marcenaro-Gutierrez (2020), e Wu (2020), que comparam o tempo de instrução e o desempenho escolar entre países mostram, de modo geral, uma modesta correlação positiva, ou mesmo nula, entre essas variáveis. Dados do Pisa indicam também que o efeito, sobre o desempenho do aluno, de uma hora adicional de aula por semana tende a ser maior nos países da Organização para a Cooperação e Desenvolvimento Econômico (OCDE) e da Europa Oriental do que nos países em desenvolvimento, devido a diferenças de produtividade do uso do tempo (LAVY, 2015). Geralmente, essas análises não eliminam diferenças entre países que podem estar relacionadas a diferenças em desempenho independentemente da jornada, mas são úteis para vislumbrar como essas variáveis relacionam-se.

Artigos que analisam países e/ou regiões e situações específicas e, portanto, não precisam lidar com diferenças regionais, também reportam relação positiva modesta, como na Suíça (CATTANEO; OGGENFUSS; WOLTER, 2017), Dinamarca (BINGLEY et al., 2018), Alemanha (MANDEL; SÜSSMUTH; 
SUNDER, 2019) e EUA (PATALL; COOPER; ALLEN, 2010; YEŞIL DAĞLI, 2018). Já os resultados para América Latina variam desde alta correlação para Colômbia até resultados negativos em Matemática para o Brasil (ALFARO; HOLLAND; EVANS, 2015).

Estudos usando estratégias de identificação mais rigorosas não trazem resultados consensuais. Na Alemanha, uma reforma que aumentou a jornada semanal em duas horas, e reduziu o número de anos para a conclusão do ano, produziu resultados ambíguos. Por um lado, houve um efeito positivo modesto no Pisa (HUEBENER; KUGER; MARCUS, 2017); por outro, houve aumento da repetência e redução do desempenho escolar em avaliações internas (HUEBENER; MARCUS, 2017). Na Dinamarca, um experimento controlado aleatorizado, que aumentou em três horas por semana, ao longo de 16 semanas, as aulas de Leitura, Escrita e Literatura teve um impacto positivo em Leitura (ANDERSEN; HUMLUM; NANDRUP, 2016). Na Itália, um programa que aumentou em $25 \%$ o tempo de aula em escolas com baixos indicadores educacionais aumentou o desempenho somente em Matemática (BATTISTIN; MERONI, 2016; MERONI; ABBIATI, 2016). Resultados positivos de intervenção, com foco em alunos de baixo desempenho, foram demonstrados em Chicago nos EUA (CORTES; GOODMAN, 2014).

Na América Latina, dois estudos mais rigorosos no Chile (BELLEI, 2009) e no México (CABRERA-HERNÁNDEZ, 2020) reportam resultados positivos e de pequena magnitude (menor que 0,1 desvio-padrão) da ampliação da jornada diária. Outros estudos não publicados vão na mesma linha, com resultados positivos e modestos, como os de Agüero (2016), Barrios Fernandez e Bovini (2019), Hincapié (2016), e Padilla-Romo (2017).

No Brasil, a maioria dos estudos ainda não foi publicada em revistas científicas, mas vale o registro. Há evidência de impacto positivo em desempenho escolar associado à reformulação do Ensino Médio em Pernambuco (ROSA et al., 2020) e de um programa piloto no município do Rio de Janeiro para o Ensino Fundamental, que implementou o turno único de sete horas diárias (CRUZ; LOUREIRO; SA, 2017). Ambos estudos destacam a importância de alterações curriculares realizadas conjuntamente ao aumento da carga horária. Já em São Paulo, segundo Aquino e Kassouf (2011), o programa de tempo integral implementado na rede estadual, aparentemente, não surtiu efeito. Da mesma forma, o programa Mais Educação, que basicamente amplia atividades extracurriculares, não obteve impactos relevantes sobre o desempenho escolar, embora haja alguma evidência de redução na evasão (GANDRA; RODRIGUES, 2018; PARENTE; PRADO, 2016). 
Em resumo, a literatura indica que, em condições normais, a ampliação da jornada não constitui uma panaceia, principalmente, levando-se em conta os custos dessa ampliação. A maioria dos efeitos positivos encontrados é modesta e depende, de forma crucial, de outros fatores, como um currículo bem estruturado.

Poucos são os estudos que analisam custos. Para o Uruguai, a evidência sugere que o investimento em tempo integral é muito menos efetivo que o de outras políticas educacionais, como transferência de renda condicionada e bolsas por mérito para meninas (ALFARO; HOLLAND; EVANS, 2015). No Brasil, estimativas mostram que a adoção do Ensino integral em 50\% das escolas públicas da Educação Básica custaria R \$ 117 bilhões por ano (ou 2\% do produto interno bruto - PIB de 2014), considerando um turno de sete horas diárias (IDADOS, 2017). Vale lembrar que, em países da OCDE, um aluno passa, em média, 7.600 horas na escola para concluir o Ensino Fundamental. No Brasil, com média de 4,5 horas por dia e 200 dias letivos, um aluno frequenta a escola por 8.100 horas para se graduar.

Essas evidências sugerem que, apesar de parecer intuitivo, não há indícios de que ampliar as horas de aula garanta ganhos de aprendizagem. Uma possível explicação reside na dificuldade de medir-se o tempo efetivo de aprendizado. Jornada diária e dias letivos indicam apenas o tempo que o aluno passa na escola, e não, necessariamente, quanto o aluno está, de fato, engajado em atividades que geram aprendizado. Os poucos estudos, como os de Bruns et al. (2014) e Kane et al. (2011), que conseguem, ainda que parcialmente, estimar esse tempo efetivo de instrução, mostram que alunos aprendem mais quando professores gastam mais tempo em sala de aula realizando atividades acadêmicas, e são capazes de mantê-los engajados por mais tempo. Nesse sentido, a capacidade do professor, novamente, assume o protagonismo.

Ademais, estudos na área da psicologia apontam os benefícios de apresentarem novas informações de forma espaçada (em contraposição à forma maciça). Assim, o indivíduo manteria o nível de atenção elevado, teria tempo para "digerir" a informação e gerar associações entre as informações novas e as antigas (JANISZEWSKI; NOEL; SAWYER, 2003). A implicação é clara: aumentar a exposição de conteúdo em um único dia pode não surtir o efeito desejado, já que sobrecarrega a memória do aluno, que se tornaria incapaz de absorver conteúdos adicionais. Ao mesmo tempo, a retenção do conteúdo anterior ficaria prejudicada por falta de prática (CEPEDA et al., 2009). Estudos realizados na Alemanha encontram efeitos negativos sobre a saúde mental dos estudantes após ampliação da jornada diária (HOFMANN; MÜHLENWEG, 2018; MARCUS et al., 2020). Na Coreia do Sul, onde a jornada pode superar 13 horas diárias, 
estudos associam períodos extensos a sintomas de depressão (LEE; LARSON, 2000). São evidências pontuais que merecem consideração.

Há também evidências sobre o papel da tecnologia no desempenho escolar. Teoricamente, a tecnologia seria uma grande aliada por sua capacidade de diagnóstico, individualização, personalização e interatividade (SOSA; MANZUOLI, 2019; TENA; GUTIÉRREZ; LLORENTE CEJUDO, 2019). Contudo, a literatura aponta resultados diversos, sendo muitos deles nulos, seja no uso de hardware (BANERJEE et al., 2007; BARRERA-OSORIO; LINDEN, 2009), seja no uso de software (BORMAN, BENSON; OVERMAN, 2009; MURALIDHARA; SINGH; GANIMIAN, 2017). Acesso a insumos também não é garantia de aprendizado. Experimentos mostram que a distribuição de computadores a alunos não está associada a maior aprendizagem (BEUERMANN et al., 2015; FAIRLIE; ROBINSON, 2013).

Essa discussão sugere que não existem saídas fáceis - seja pela ampliação do horário escolar, seja pela adoção de tecnologias. Assim, não parece ser o momento de improvisar ou de experimentar ideias não testadas em larga escala, mas, sim, de tomar decisões baseadas em evidências. Mesmo porque, é provável que haja várias ondas e momentos pós-pandemia.

A próxima seção apresenta o que a literatura tem apontado como estratégias eficazes e eficientes para melhorar a aprendizagem dos alunos. Novamente, diz respeito a programas implementados em condições normais e, portanto, não seriam diretamente aplicáveis ao caso brasileiro pós-pandemia sem o devido cuidado.

\section{Estratégias potencialmente eficazes face às limitações estruturais}

Desde a publicação do relatório Coleman (COLEMAN et al., 1966), inúmeros artigos avaliaram fatores associados ao desempenho dos alunos, sejam eles externos ou não à escola. Dado que o interesse, aqui, recai sobre estratégias de Ensino e ações que podem ser adotadas pelas escolas para mitigar os efeitos da quarentena, não analisaremos políticas que impactam variáveis externas, como nível socioeconômico.

Os fatores e as estratégias de Ensino que possuem efeitos robustos e bem documentados na literatura dependem basicamente da qualidade do professor. Um dos indicadores é a clareza, isto é, a capacidade do professor de comunicarse bem e passar o conteúdo de forma organizada. Isso tem sido estudado sob 
diversos tópicos, classificados por John Hattie como teacher clarity (TITSWORTH et al., 2015) e conceptual change (ARMAGAN; KESKIN; AKIN, 2010), por exemplo. Outros analisam a capacidade cognitiva e socioemocional. Há evidências consolidadas acerca da dificuldade de atrair os melhores alunos para tornaremse professores, portanto não é algo com que se possa contar no curto prazo (LOUZANO et al., 2010). De acordo com dados do Instituto Nacional de Estudos e Pesquisas Educacionais Anísio Teixeira (Inep), o Indicador de Diferença entre os Desempenhos (IDD), a média no Exame Nacional do Ensino Médio (Enem) dos professores formados mais recentemente está abaixo da média geral no exame (dados compilados pelos autores - disponíveis, a pedido).

Se no Ensino presencial o papel do professor é fundamental, no Ensino remoto isso, provavelmente, também seria o caso, desde que este tivesse familiaridade com tecnologias e técnicas eficazes de Ensino a distância. No caso concreto, isso resultará na manutenção ou ampliação das desigualdades, dada a impossibilidade de desenvolver estratégias mais genéricas e robustas, no curto prazo, para suprir as carências no setor público.

Analogamente, a habilidade dos pais em ensinar é limitada, principalmente em se tratando de conteúdos específicos das séries mais avançadas e de interações e de estímulos realizados na primeira infância, que possuem uma curta janela temporal para serem realizados (AVVISATI et al., 2014; POWELL-SMITH et al., 2000). No caso de crianças muito pequenas, muito pode ser feito em casa pelos pais - e efetivamente o é, sobretudo, por famílias de classes sociais mais favorecidas, em tempos normais. Em tempos de pandemia, as pressões sobre as famílias, possivelmente, constituirão oportunidades para desenvolver habilidades, como o estabelecimento de rotinas e de resiliência, mas dificilmente constituem oportunidades favoráveis para aprender e adotar novas formas de interação pelos pais, especialmente em ambientes mais carentes.

Ademais, dada as limitações financeiras, não é possível, na maioria dos casos, a contratação de tutores para acompanhar os alunos em atividades direcionadas. Há evidências de que tutorias durante as férias podem ter efeitos positivos, ainda que os resultados não sejam taxativos (FRYER, 2014; SCHUELER et al., 2017). Uma opção para as famílias, em tempos sem aulas e para qualquer faixa etária, é a leitura. A distribuição de livros antes das férias escolares gera impactos positivos em Leitura (ALLINGTON et al., 2010; KIM; WHITE, 2008). Para os menores de seis anos, a leitura de livros com os pais tem suporte na literatura, beneficiando aquisição de vocabulário, aumentando a frequência de boas interações entre pais e filhos e até mesmo reduzindo a violência doméstica (WEISLEDER et al., 2016). 
Não menos importante é a questão da desigualdade socioeconômica brasileira no contexto educacional. A literatura tem mostrado que em qualquer país é difícil reduzir desigualdades entre grupos de alunos ao longo das séries escolares (HECKMAN; MASTEROV, 2007; HIPPEL; HAMROCK, 2019). A diferença de vocabulário entre crianças pobres e ricas já é substancial aos dois anos de idade, segundo Hart e Hisley (1995). No entanto, a partir da entrada na Educação formal, entre cinco e sete anos de idade, dependendo do país, as diferenças permanecem relativamente estáveis ao longo dos anos, incluindo o período de férias escolares (HIPPEL; HAMROCK, 2019). Essas evidências sugerem que as escolas têm limitações para reduzir desigualdades, embora possam realizar iniciativas direcionadas a determinados públicos-alvo, como tutorias intensivas de alta qualidade, por exemplo, para auxiliar alunos com maiores dificuldades.

Isto posto, a maioria das intervenções possuem efeitos heterogêneos, impactando mais um determinado grupo do que outro. O programa de tutoria intensiva, em Nova York, por exemplo, impactou mais os alunos negros e latinos do que os brancos, e mais os negros do que os latinos, mas não impactou mais os mais pobres (FRYER JUNIOR; HOWARD-NOVECK, 2020). Já o programa que ofereceu incentivo e treinamento aos pais para que participassem mais da vida escolar dos filhos, realizado em Chicago, resultou em maiores ganhos para brancos e hispânicos e para os alunos com maior habilidade não cognitiva medida antes do início da intervenção (FRYER JUNIOR; LEVITT; LIST, 2015). Seria o caso de descontinuar tais programas porque geraram resultados diferentes para diferentes grupos? Se a resposta for positiva, no limite, isso implicaria descontinuar todo e qualquer programa elaborado para dar apoio adicional a quem mais precisa, pois, certamente, haverá um grupo de alunos que conseguirá beneficiar-se mais que outros.

A pandemia também trouxe à tona as limitações do Ensino remoto, que pode apresentar-se de distintas formas, como aulas on line, por correspondência, por televisão ou videoconferências. Estudos que comparam o Ensino em sala de sala com o Ensino a distância, principalmente, on line, apresentam uma grande heterogeneidade (BERNARD et al., 2004; CAVANAUGH et al., 2004; MEANS et al., 2009; MORGAN, 2015). Essa variação passa pela qualidade dos programas e de sua implementação, especialmente, os problemas de frequência dos alunos, monitoramento do tempo gasto on line (MORGAN, 2015; QUEEN; LEWIS, 2011) e não ter o mesmo cuidado utilizado em abordagens tradicionais (CAVANAUGH et al., 2004). Um ambiente que combine o Ensino presencial com orientações on line parece ser o que produz melhores resultados, se bem organizado e estruturado (MEANS et al., 2009; O'DWYER; CAREI; KLEIMAN, 2007; SUN; LIN; YU, 2008). A evidência é contundente e oportuna: não é a disponibilidade de equipamentos e o acesso à internet que fariam diferença, mas sim, como a tecnologia é inserida no contexto escolar. 


\section{O que poderia funcionar - com base nas circunstâncias e nas evidências}

A seção anterior tornou evidente que as estratégias mais promissoras - inclusive baseadas em tecnologias - pressupõem um conjunto de condições que estão longe do alcance da esmagadora maioria das redes públicas de Ensino e, notadamente, as limitações associadas aos professores, sua falta de experiência com tecnologias, bem como as limitações de conectividade, de infraestrutura e de interação ao ambiente domiciliar. Resta, portanto, examinar um segundo conjunto de estratégias que poderiam ser eficazes em nosso contexto.

Seguindo os mesmos critérios utilizados na revisão da literatura anterior, surge como mais promissor um conjunto estratégias que faça melhor uso do tempo disponível no restante do ano letivo, e que incluem: (a) alfabetização usando métodos fônicos, (b) leitura; (c) avaliação diagnóstica; (d) Ensino estruturado; (e) tutorias; (f) dever de casa e (g) garantia da frequência escolar.

A maioria das evidências sobre alfabetização usando métodos fônicos são positivas. Desde a década de 1990, essa questão ficou bem estabelecida na literatura, especialmente, a partir do estudo de Adams (1994) e das publicações do National Reading Panel (2000) e do National Institute for Literacy (2008). Conforme Fryer e Howard-Noveck (2020), também há evidência positiva sobre a leitura acompanhada de tutoria adequada, bem como a leitura interativa para crianças - que funciona, independentemente, do nível de escolaridade e de alfabetização dos pais (SÉNÉCHAL; LEFEVRE, 2002; WEISLEDER et al., 2016; ZUCKERMAN; AUGUSTYN, 2011).

Evidências sobre avaliação diagnóstica sugerem que sua eficácia está mais diretamente associada à implementação de estratégias sistematizadas (reformas educacionais ou curriculares) em escolas e redes escolares, conforme Murnane, Sharkey e Boudett (2005). A utilidade do diagnóstico está vinculada à disponibilidade de meios e à capacidade de implementar estratégias eficazes para superar as dificuldades dos alunos. Isso também explica, em parte, o sucesso de programas de tutoria em pequenos grupos - um tutor experiente é capaz de calibrar as intervenções às dificuldades de cada aluno.

A extensa literatura sobre Ensino estruturado (direct instruction) sugere duas orientações complementares: estratégias didáticas mais eficazes e compensação de deficiências dos professores com materiais de Ensino de melhor qualidade. Uma meta-análise, cobrindo mais de 300 experimentos realizados nos últimos 50 anos, reportou resultados positivos em Leitura e Matemática, além de efeitos 
positivos também em outras dimensões (STOCKARD et al., 2018). Além disso, Piper et al. (2018) explicam como a eficácia dessa abordagem requer uma mistura de ingredientes implementados de maneira articulada e consistente. Evidências mais recentes dessa estratégia foram observadas no Quênia, como alternativa para "acelerar" perdas decorrentes de um período prolongado de suspensão de aulas (WILICHOWSKI et al., 2020). Como em qualquer intervenção, não é o modelo "Ensino estruturado" que funciona em si, mas o conjunto de seus elementos, inclusive e especialmente, a qualidade dos materiais e a adequação das estratégias pedagógicas à condição dos professores.

Programas de tutoria realizados no contraturno, em pequenos grupos homogêneos, têm-se mostrado mais eficazes justamente para alunos com maiores dificuldades (FRYER JUNIOR; HOWARD-NOVECK, 2020). Entretanto, os programas mais eficazes são intensivos de recursos humanos qualificados. Isso limita sua adoção em momentos de restrição orçamentária e sugere seu uso em alunos com maiores lacunas.

Focalizar o dever de casa constitui alternativa de baixo custo, que pode ser combinada com tutoria, permitindo ao aluno fixar conceitos e praticar exercícios sem atropelar a aprendizagem. Entretanto, as evidências nesse caso são menos robustas. Embora Roschelle et al. (2016) afirmem que haja dados experimentais, muitos estudos em meta-análises não foram publicados ou apresentam impactos positivos relativamente pequenos, como os de e Fan et al. (2017). O efeito pode depender da qualidade da tarefa demandada pelo professor e do envolvimento dos pais na lição de casa (controle e suporte), que pode impactar a motivação e o comportamento do aluno em relação à lição de casa. Alunos em turmas com tarefas bem selecionadas e desafiadoras aprendem mais que seus pares (DETTMERS et al., 2010). Alunos com pais menos controladores, que exercem menos pressão sobre a lição de casa, e mais apoiadores, que dão mais assistência às tarefas, obtém melhores desempenhos (NÚÑEZ et al., 2015; VAN VOORHIS, 2011). Ademais, o tempo ótimo gasto em dever de casa pode variar entre as etapas de Ensino (COOPER et al., 2006). Castro et al. (1978) já haviam observado que quanto melhor o nível do Ensino, menor o tempo gasto em deveres de casa. Dado o baixo custo e as vantagens associadas ao dever de casa, como formação de hábitos de estudo, responsabilidade, promoção da autonomia, prática espaçada e memorização, trata-se de alternativas a serem consideradas.

Finalmente, com base nos dados do National Forum on Education Statistics (AR2009), cabe lembrar a importância de investir na frequência escolar, especialmente dos alunos de maior risco, pois trata-se de estratégia de custo zero e fortemente associada à deserção escolar. 


\section{Para concluir - o que aprendemos com evidências}

As evidências aqui revistas constituem um corpo sólido de fatos científicos bem estabelecidos e que podem orientar políticas e práticas de intervenção no contexto pós-pandemia. É possível que haja perdas decorrentes da interrupção de aulas e que as perdas sejam maiores em determinados níveis de Ensino, disciplinas - especialmente, Matemática -, e grupos menos favorecidos. A longo prazo, no entanto, essas perdas tendem a ser recuperadas e, mediante intervenções corretas, as diferenças entre grupos sociais podem ser atenuadas com a adoção de estratégias adequadas.

As saídas consideradas mais óbvias para o período pós-pandemia - como o Ensino remoto, o uso de tecnologias e o aumento da carga horária - dificilmente constituem soluções robustas para enfrentar as consequências da interrupção do calendário escolar. A literatura revista sugere que, embora estejam sendo incorporadas ao dia a dia da escola com maior intensidade, essas três opções dificilmente contêm os elementos para ajudar na recuperação dos alunos mais prejudicados.

Diante do contexto educacional do país, as saídas mais solidamente fundamentadas na literatura incluem, em primeiro lugar, um diagnóstico dos alunos como base para a retomada dos programas de Ensino. E, a partir daí, intervenções robustas e promissoras que incluem, do lado pedagógico, o Ensino estruturado, o uso de métodos adequados de alfabetização, o uso estratégico dos deveres de casa e de programas de leitura. O melhor uso do tempo consiste na redução do absenteísmo e, para os alunos com mais dificuldade, programas intensivos de tutoria em pequenos grupos.

Sobretudo, eventos como a pandemia desnudam a fonte e a origem das desigualdades, cuja atenuação requer políticas intensivas para a Primeira Infância e atenção especial para os alunos nos primeiros anos escolares. 


\section{Covid-19 and back to school: listening to evidence}

\section{Abstract}

Paralyzed by panic induced by the Covid-19 pandemic, school closures may impact student learning. This paper examines situations of school closures, use of time and impact of technologies on student achievement. Investigation of the evidence suggests that timeintensive strategies or the use of technologies are not promising. More effective ways are associated with diagnoses, direct instruction suited to the profile of teachers, better use of time, judicious use of homework, reduced absenteeism and intensive high-quality tutoring focused on the most at-risk students.

Keywords: Covid-19. Interruption of School Calendar. Evidence-based Education.

\section{Covid-19 y el regreso a las clases: escuchando las evidencias}

\section{Resumen}

Paralizadas por el pánico resultante de la pandemia de Covid-19, el cierre de las escuelas podrá tener consecuencias para el aprendizaje de los estudiantes. El artículo examina situaciones de parálisis, uso del tiempo e impacto de las tecnologías en el rendimiento escolar. El examen de las evidencias sugiere que las estrategias que requieren mucho tiempo o el uso de la tecnología no son prometedoras. Se asociarían caminos más prometedores con diagnósticos, intervenciones estructuradas más adecuadas para el perfil de los maestros, mejor uso del tiempo, uso juicioso de la tarea, reducción del ausentismo y tutoría intensiva de alta calidad centrada en los estudiantes más en riesgo.

Palabras-clave: Covid-19. Interrupción del Calendario Escolar. Educación Basada en Evidencias. 


\section{REFERÊNCIAS}

ADAMS, M. J. Benning to read. thinking and learning about print. Cambridge: MIT Press, 1994.

AGÜERO, J. M. Evaluación de impacto de la jornada escolar completa. Conecticut: Universidad de Connecticut, 2016.

ALFARO, P.; HOLLAND, P. A.; EVANS, D. Extending the school day in Latin America and the Caribbean. [Washington, DC]: World Bank Group, 2015. (Policy Research Working Paper, n. 7309).

ALLINGTON, R. L. et al. Addressing summer reading setback among economically disadvantaged elementary students. Reading Psychology, London, v. 31, n. 5, p. 411-427, Sep. 2010. https://doi.org/10.1080/02702711.2010.505165

ANDERSEN, S. C.; HUMLUM, M. K.; NANDRUP, A. B. Increasing instruction time in school does increase learning. Proceedings of the National Academy of Sciences of the United States of America, Washington, DC, v. 113, n. 27, p. 7481-7484, June 2016. https://doi.org/10.1073/pnas.1516686113

AQUINO, J. M.; KASSOUF, A. L. A ampliação da jornada escolar melhora o desempenho acadêmico dos estudantes? Uma avaliação do programa Escola de Tempo Integral da rede pública do Estado de São Paulo. 2011. Disponível em: http://reap.org.br/wp-content/uploads/2011/11/013-AAmplia\%C3\%A7\%C3\%A3o-da-Jornada-Escolar-melhora-o-Desempenho. pdf. Acesso em: 20 abr. 2020.

ARMAGAN, F. O.; KESKIN, M. O; AKIN, S.B. Effectiveness of conceptual change texts: a meta analysis. Procedia - Social and Behavioral Sciences, New York, v. 9, p. 1574-1579, Dec. 2010. https://doi.org/10.1016/j.sbspro.2010.12.367

ATTEBERRY, A., MCEACHIN, A. School's out: the role of summers in understanding achievement disparities. Providence:Annenberg Institute at Brown University, 2020. (EdWorkingPaper, n. 19-82).

AVVISATI, F. et al. Getting parents involved: a field experiment in deprived schools. The Review of Economic Studies, Oxford, v. 81, n. 1, p. 57-83, Jan. 2014. https://doi.org/10.1093/restud/rdt027

BANERJEE, A. V. et al. Remedying education: evidence from two randomized experiments in India. The Quarterly Journal of Economics, Oxford, v. 122, n. 3, p. 1235-1264, Feb. 2007. https://doi.org/10.1162/qjec.122.3.1235 
BARRERA-OSORIO, F.; LINDEN, L. L. The use and misuse of computers in education: evidence from a randomized controlled trial of a language arts program. Cambridge: Abdul Latif Jameel Poverty Action Lab, 2009.

BARRIOS FERNÁNDEZ, A.; BOVINI, G. Itts time to learn: understanding the differences in returns to instruction time. SSRN Electronic Journal, Rochester, Aug. 2018. https://doi.org/10.2139/ssrn.2932359

BATTISTIN, E.; MERONI, E. C. Should we increase instruction time in low achieving schools? Evidence from Southern Italy. Economics of Education Review, Cambridge, v. 55, p. 39-56, Dec. 2016. https://doi.org/10.1016/j.econedurev.2016.08.003

BELLEI, C. Does lengthening the school day increase students' academic achievement? Results from a natural experiment in Chile. Economics of Education Review, Cambridge, v. 28, n. 5, p. 629-640, Oct. 2009. https://doi. org/10.1016/j.econedurev.2009.01.008

BELOT, M., WEBBINK, D. Do teacher strikes harm educational attainment of students? Labour, Oxford, v. 24, n. 4, p. 391-406, Nov. 2010. https://doi. org/10.1111/j.1467-9914.2010.00494.x

BENSON, J.; BORMAN, G. Family, neighborhood, and school settings across seasons: when do socioeconomic context and racial composition matter for the reading achievement growth of young children? Teachers College Record, New York, v. 112, n. 5, p. 1338-1390, May 2010. Disponível em: https://www. researchgate.net/publication/285719563_Family_Neighborhood_and_School_ Settings_Across_Seasons_When_Do_Socioeconomic_Context_and_Racial_ Composition_Matter_for_the_Reading_Achievement_Growth_of_Young Children. Acesso em: 29 abr. 2020.

BERNARD, R. M. et al. How does distance education compare with classroom instruction? A meta-analysis of the empirical literature. Review of Educational Research, Washington, DC, v. 74, n. 3, p. 379-439, Sep. 2004. https://doi.org/10.3102/00346543074003379

BEUERMANN, D. W. et al. One laptop per child at home: short-term impacts from a randomized experiment in Peru. American Economic Journal: Applied Economics, London, v. 7, n. 2, p. 53-80, Apr. 2015. https://doi.org/10.1257/app.20130267

BINGLEY, P. et al. The timing of instruction time: accumulated hours, timing and pupil achievement. Copenhagen: The Rockwool Foundation Research Unit, 2018. (Study Paper, n. 131). 
BORMAN, G. D.; BENSON, J. G.; OVERMAN, L. A randomized field trial of the Fast ForWord Language computer-based training program. Educational Evaluation and Policy Analysis, Washington, DC, v. 31, n. 1, p. 82-106, Mar. 2009. https://doi.org/10.3102/0162373708328519

BRUNS, B. et al. Inside the classroom in Latin America and the Caribbean. In: BRUNS, B., LUQUE, J. (orgs.) Great teachers: how to raise student learning in latin america and the caribbean. Washington, DC: The World Bank, 2014. p. 97-137.

CABRERA-HERNÁNDEZ, F. Does lengthening the school day increase school value added? Evidence from a mid-income country. The Journal of Development Studies, v. 56, n. 2, p. 314-335, 2020. https://doi.org/10.1080/00220388.2018.1563680

CASTRO, C. M. et al. Custos e determinantes da educação: o caso de Brasília. Rio de Janeiro: SEC/DF/ECIEL, 1978.

CATTANEO, M. A.; OGGENFUSS, C.; WOLTER, S. C. The more, the better? The impact of instructional time on student performance. Education Economics, London, v. 25, n. 5, p. 433-445, 2017. https://doi.org/10.1080/096 45292.2017.1315055

CAVANAUGH, C. S. et al. The effects of distance education on K-12 student outcomes: a meta-analysis. Naperville: Learning Point Associates, 2004.

CEPEDA, N. J et al. Optimizing distributed practice: theoretical analysis and practical implications. Experimental Psychology, Göttingen, v. 56, n. 4, p. 236-246, 2009. https://doi.org/10.1027/1618-3169.56.4.236

COLEMAN, J. S. et al. Equality of education opportunity study. Washington, DC: US Government Printing Office, 1966.

COOPER, H. et al. Does homework improve academic achievement? a synthesis of research, 1987-2003. Review of Educational Research, Washington, DC, v. 76, n. 1, p. 1-62, Mar. 2006. https://doi.org/10.3102/00346543076001001

CORTES, K. E.; GOODMAN, J. S. Ability-tracking, instructional time, and better pedagogy: the effect of double-dose algebra on student achievement. American Economic Review, Nashville, v. 104, n. 5, p. 400-405, 2014. https://doi.org/10.1257/aer.104.5.400

CRUZ, T.; LOUREIRO, A.; SA, E. Full-time teachers, students, and curriculum: the single shift model in Rio de Janeiro. Washington, DC: World Bank Group, 2017. (Policy Research Working Paper, n. 8086). 
DETTMERS, S. et al. Homework works if homework quality is high: using multilevel modeling to predict the development of achievement in mathematics. Journal of Educational Psychology, Washington, DC, v. 102, n. 2, p. 467-482, May 2010. https://doi.org/10.1037/a0018453

FAIRLIE, R.; ROBINSON, J. Experimental evidence on the effects of home computers on academic achievement among schoolchildren. American Economic Journal: Applied Economics, Nashville, v. 5, n. 3, p. 211-240, July 2013. https://doi.org/10.1257/app.5.3.211

FAN, H. et al. Homework and students' achievement in math and science: A 30-year meta-analysis, 1986-2015. Educational Research Review, [s. 1.], v. 20, p. 35-54, Feb. 2017. https://doi.org/10.1016/j.edurev.2016.11.003

FRYER, R. G. Injecting charter school best practices into traditional public schools: evidence from field experiments. Quarterly Journal of Economics, Oxford, v. 129, n. 3, p. 1355-1407, Sep. 2014. https://doi.org/10.1093/qje/ qju011

FRYER JUNIOR, R. G.; HOWARD-NOVECK, M. High-dosage tutoring and reading achievement: evidence from New York City. Journal of Labor Economics, Chicago, v. 38, n. 2, p. 421-452, Apr. 2020. Disponível em: https:// www.journals.uchicago.edu/doi/abs/10.1086/705882?af=R\&mobileUi=0. Acesso em: 29 abr. 2020.

FRYER JUNIOR, R. G.; LEVITT, S.D.; LIST, J. A. Parental incentives and early childhood achievement: a field experiment in Chicago heights. Cambridge: National Bureau of Economic Research, 2015. (NBER Workin Paper Series, n. 21477).

GANDRA, J. M. F. V.; RODRIGUES, C. T. O impacto da educação em tempo integral no desempenho escolar: uma avaliação do programa Mais Educação Encontro Nacional de Estudos Populacionais. In: ENCONTRO NACIONAL DE ESTUDOS POPULACIONAIS, 21., 2018., Poços de Caldas. Anais[...]. Poços de Caldas: Abep, 2018. Disponível em: http://www. abep.org.br/publicacoes/index.php/anais/article/view/3075/2937. Acesso em: 29 abr. 2020.

HART, B.; RISLEY, T. R. Meaningful differences in the everyday experience of young American children. Baltimore: Paul H Brookes, 1995.

HATTIE, J. Para uma lista de tópicos já estudados. Disponível em: http:// www.visiblelearningmetax.com/Influences. Acesso em: 20 abr. 2020. 
HECKMAN, J. J.; MASTEROV, D. V. The productivity argument for investing in young children. Review of Agricultural Economics, Oxford, v. 29, n. 3, p. 446-493, Feb. 2007. https://doi.org/10.2307/4624854

HINCAPIÉ, D. Do longer school days improve student achievement? Evidence from Colombia. [S. 1.]: Inter-American Development Bank, 2016. (IDB Working Paper Series, n. IDB-WP-679).

HIPPEL, P. T.; HAMROCK, C. Do test score gaps grow before, during, or between the school years? measurement artifacts and what we can know in spite of them. Sociological Science, [s. 1.], v. 6, p. 43-80, Jan. 2019. https://doi. org/10.15195/v6.a3

HIPPEL, P. T.; WORKMAN, J.; DOWNEY, D. B. Inequality in reading and math skills forms mainly before kindergarten: a replication, and partial correction, of "Are schools the great equalizer?". Sociology of Education, New York, v. 91, n. 4, p. 323-357, Sep. 2018. https://doi. org/10.1177/0038040718801760

HOFMANN, S.; MÜHLENWEG, A. Learning intensity effects in students' mental and physical health: evidence from a large-scale natural experiment in Germany. Economics of Education Review, Cambridge, v. 67, p. 216-234, Dec. 2018. https://doi.org/10.1016/j.econedurev.2018.10.001

HUEBENER, M.; KUGER, S.; MARCUS, J. Increased instruction hours and the widening gap in student performance. Labour Economics, Amsterdam, v. 47, p. 15-34, Aug. 2017. https://doi.org/10.1016/j.labeco.2017.04.007

HUEBENER, M.; MARCUS, J. Compressing instruction time into fewer years of schooling and the impact on student performance. Economics of Education Review, Cambridge, v. 58, p. 1-14, June 2017. https://doi. org/10.1016/j.econedurev.2017.03.003

IDADOS. Quanto custa o Plano Nacional de Educação (2014-2024)? Rio de Janeiro: IDados, 2017.

JANISZEWSKI, C.; NOEL, H.; SAWYER, A. G. A meta-analysis of the spacing effect in verbal learning: implications for research on advertising repetition and consumer memory. Journal of Consumer Research, Gainesville, v. 30, n. 1, p. 138-149, Feb. 2003. https://doi.org/10.1086/374692

KANE, T. J. et al. Identifying effective classroom practices using student achievement data. Journal of Human Resources, Gainesville, v. 46, n. 
3, p. 587-613, Summer 2011. Disponível em: https://www.jstor.org/ stable/41304833?seq=1. Acesso em: 20 abr. 2020.

KIM, J. S.; WHITE, T. G. Scaffolding voluntary summer reading for children in grades 3 to 5: an experimental study. Scientific Studies of Reading, Mahwah, v. 12, n. 1, p. 1-23, Jan. 2008. https://doi. org/10.1080/10888430701746849

KUHFELD, M. Surprising new evidence on summer learning loss. Phi Delta Kappan, Bloomington, v. 101, n. 1, p. 25-29, Aug. 2019. https://doi. org/10.1177/0031721719871560

KUHFELD, M.; SOLAND, J. The learning curve: revisiting the assumption of linear growth across the school year. Providence: Annenberg Institute at Brown University, 2020. (EdWorking Paper, n. 20-214).

LAVY, V. Do differences in Schools' instruction time explain international achievement gaps? Evidence from developed and developing countries. Economic Journal, London, v. 125, n. 588, p. 397-424, Nov. 2015. https://doi.org/10.1111/ecoj.12233

LEE, M.; LARSON, R. The Korean 'examination hell': long hours of studying, distress, and depression. Journal of Youth and Adolescence, New York, v. 29, n. 2, p. 249-271, Apr. 2000. https://doi. org/10.1023/A:1005160717081

LONG, D. A. Cross-national educational inequalities and opportunities to learn: conflicting views of instructional time. Educational Policy, Thousad Oaks, v. 28, n. 3, p. 351-392, May 2013. https://doi. org/10.1177/0895904812465108

LOPEZ-AGUDO, L. A.; MARCENARO-GUTIERREZ, O. D. Instruction time and students academic achievement: a cross-country comparison. Compare, Oxford, Mar. 2020. https10.1080/03057925.2020.1737919

LOUZANO, P. et al. Quem quer ser professor? Atratividade, seleção e formação do docente no Brasil. Estudos em Avaliação Educacional, São Paulo, v. 21, n. 47, p. 543-568, 2010. https://doi.org/10.18222/ eae214720102463

MANDEL, P.; SÜSSMUTH, B.; SUNDER, M. Cumulative instructional time and student achievement. Education Economics, London, v. 27, n. 1, p. 20-34, 2019. https://doi.org/10.1080/09645292.2018.1512559 
MARCUS, J. et al. Increased instruction time and stress-related health problems among school children. Journal of Health Economics, Amsterdam, v, 70, 102256, Mar. 2020. https://doi.org/10.1016/j.jhealeco.2019.102256

MCMULLEN, S. C.; ROUSE, K. E. The impact of year-round schooling on academic achievement: evidence from mandatory school calendar conversions. American Economic Journal: Economic Policy, Nashville, v. 4, n. 4, p. 230-252, Nov. 2012. Disponível em: https://www.aeaweb.org/ articles?id=10.1257/pol.4.4.230. Acesso em: 20 abr. 2020

MEANS, B. et al. Evaluation of evidence-based practices in online learning: a meta-analysis and review of online learning studies. Washington, DC: U.S. Department of Education, 2009.

MERONI, E. C.; ABBIATI, G. How do students react to longer instruction time? Evidence from Italy. Education Economics, London, v. 24, n. 6, p. 592611, Jan. 2016. https://doi.org/10.1080/09645292.2015.1122742

MEYERS, K.; THOMASSON, M. A. Paralyzed by panic: measuring the effect of school closures during the 1916 polio pandemic on educational attainment. Cambridge: National Bureau of Economic Research, 2017. (NBER Working Paper Series, n. 23890).

MORGAN, H. Online instruction and virtual schools for middle and high school students: twenty-first-century fads or progressive teaching methods for today's pupils? The Clearing House, Menasha, v. 88, n. 2, p. 72-76, 2015. https://doi.org/10.1080/00098655.2015.1007909

MURALIDHARAN, K.; SINGH, A.; GANIMIAN, A. J. Disrupting education? Experimental evidence on technology-aided instruction in India. American Economic Review, Nashville, v. 109, n. 4, p. 1426-60, Apr. 2019. https://doi.org/10.1257/aer.20171112

MURNANE, R. J.; SHARKEY, N. S.; BOUDETT, K. P. Using studentassessment results to improve instruction: lessons from a workshop. Journal of Education for Students Placed at Risk, London, v. 10, n. 3, p. 269-280, July 2005. https://doi.org/10.1207/s15327671espr1003_3

NATIONAL FORUM ON EDUCATION STATISTICS. Every school day counts: the forum guide to collecting and using attendance data. Washington, DC, 2009.

NATIONAL INSTITUTE OF CHILD HEALTH AND HUMAN DEVELOPMENT. Report of the national reading panel: teaching children to read. Washington, DC, 2000. 
NATIONAL INSTITUTE FOR LITERACY. Developing early literacy: report of the national early literacy panel. Washington, DC, 2008

NATIONAL READING PANEL. Teaching children to read: an evidencebased assessment of the scientific research literature on reading and its implications for reading instruction. Rockville: National Institute of Child Health and Human Development, 2000.

NÚÑEZ, J. C. et al. Relationships between perceived parental involvement in homework, student homework behaviors, and academic achievement: differences among elementary, junior high, and high school students. Metacognition and Learning, New York, v. 10, n. 3, p. 375-406, Feb. 2015. https://doi.org/10.1007/s11409-015-9135-5

O'DWYER, L. M.; CAREI, R.; KLEIMAN, G. A study of the effectiveness of the louisiana algebra i online course. Journal of Research on Technology in Education, [s. 1.], v. 39, n. 3, p. 289-306, Jan. 2007. https://doi.org/10.1080/15 391523.2007.10782484

PARENTE, C.; PRADO, R. Programa mais educação: análise das produções acadêmicas. Revista de Humanidades, Fortaleza, v. 3, n. 2, p. 563-583, jul./ dez. 2016. https://doi.org/10.5020/23180714.2016.31.2.563-583

PADILLA-ROMO, M. The short and long run effects of full-time schools on academic performance. College Station: A\&M University, 2017.

PATALL, E. A.; COOPER, H.; ALLEN, A. B. Extending the school day or school year: a systematic review of research (1985-2009). Review of Educational Research, Washington, DC, v. 80, n. 3, p. 401-436, Sep. 2010. https://doi.org/10.3102/0034654310377086

PATTON, K. L. S.; RESCHLY, A. L. Using curriculum-based measurement to examine summer learning loss. Psychology in the Schools, Brandon, v. 50, n. 7, p. 738-753, Aug. 2013. https://doi.org/10.1002/pits.21704

PIPER, B. et al. Identifying the essential ingredients to literacy and numeracy improvement: teacher professional development and coaching, student textbooks, and structured teachers' guides. World Development, Oxford, v. 106, p. 324-336, June 2018. https://doi.org/10.1016/j. worlddev.2018.01.018

POWELL-SMITH, K. A. et al. Parent tutoring in reading using literature and curriculum materials: impact on student reading achievement. School 
Psychology Review, Cuyahoga Falls, v. 29, n. 1, p. 5-27, Jan. 2000. Disponível em: https://www.tandfonline.com/doi/abs/10.1080/02796015.2000.12085995. Acesso em: 20 abr. 2020.

QUEEN, B., LEWIS, L. Distance education courses for public elementary and secondary school students: 2009-10. Washington, DC: U.S. Department of Education, National Center for Education Statistics, 2011.

QUINN, D. M. Black-white summer learning gaps: interpreting the variability of estimates across representations. Educational Evaluation and Policy Analysis, Washington, DC, v. 37, n. 1, p. 50-69, 2015. https://doi. org/10.3102/0162373714534522

QUINN, D. M.; LE, Q. T. Are we trending to more or less between-group achievement inequality over the school year and summer? AERA Open, Washington, DC, Dec. 2018. https://doi.org/10.1177/2332858418819995

RAMBO-HERNANDEZ, K. E.; MCCOACH, D. B. High-achieving and average students' reading growth: contrasting school and summer trajectories. The Journal of Educational Research, Madison, v. 108, n. 2, p. 112-129, Nov. 2014. https://doi.org/10.1080/00220671.2013.850398

ROSA, L. et al. The effects of public high school subsidies on student test scores: the case of a full-day high school program in Pernambuco, Brazil. 2020. Disponível em: https://drive.google.com/file/d/1X_gOy1Zq40VHR-MLusey1YIIshF7fwU/view. Acesso em: 29 abr. 2020.

ROSCHELLE, J. et al. Online mathematics homework increases student achievement. AERA Open, Washinton, DC, Oct. 2016. https://doi. org $/ 10.1177 / 2332858416673968$

SACERDOTE, B. When the saints go marching out: long-term outcomes for student evacuees from hurricanes Katrina and Rita. American Economic Journal: Applied Economics, Nashville, v. 4, n. 1, p. 109-135, Jan. 2012. https://doi.org/10.1257/app.4.1.109

SÉNÉCHAL, M.; LEFEBRE, J.-A. Parental involvement in the development of children's reading skill: a five-year longitudinal study. Child Development, Malden, v. 73, n. 2, p. 445-460, 2002. https://doi. org/10.1111/1467-8624.00417

SCHUELER, B. et al. Can states take over and turn around school districts? Evidence from Lawrence, Massachusetts. Educational Evaluation and 
Policy Analysis, Washingon, DC, v. 39, n. 2, p. 31-32, Jan. 2017. https://doi. org/10.3102/0162373716685824

SOSA, O., MANZUOLI, C. Models for the pedagogical integration of information and communication technologies: a literature review. Ensaio: Avaliação e Políticas Públicas em Educação, Rio de Janeiro, v. 27, n. 102, p. 129-156, jan./mar. 2019. http://dx.doi.org/10.1590/s0104-40362018002701720

STOCKARD, J. et al. The effectiveness of direct instruction curricula: A meta-analysis of a half century of research. Review of Educational Research, Washington, DC, v. 88, n. 4, p. 479-507, Jan. 2018. https://doi. org/10.3102/0034654317751919

SUN, K.; LIN, Y.; YU, C. A study on learning effect among different learning styles in a Web-based lab of science for elementary school students. Computers \& Education, New York, v. 50, n. 4, p. 1411-1422, May 2008. https://doi.org/10.1016/j.compedu.2007.01.003

TENA, R.; GUTIÉRREZ, M. P.; LLORENTE CEJUDO, M. C. Technology use habits of children under six years of age at home. Ensaio: Avaliação e Políticas Públicas em Educação, Rio de Janeiro, v. 27, n. 103, p. 340-362, abr./jun. 2019. https://doi.org/10.1590/s0104-40362019002701752

TITSWORTH, T. et al. Two meta-analyses exploring the relationship between teacher clarity and student learning. Communication Education, Abingdon, v. 64, n. 4, p. 385-418, June 2015. https://doi.org/10.1080/03634523.2015.1041 998

UNESCO. COVID-19 impact on education. Disponível em: https://en.unesco. org/covid19/educationresponse. Acesso em: 2 maio 2020.

VALE, C. et al. Growth in literacy and numeracy achievement: evidence and explanations of a summer slowdown in low socio-economic schools. The Australian Educational Researcher, North Ryde, v. 40, p. 1-25, 2013. https:// doi.org/10.1007/s13384-012-0065-9

VAN VOORHIS, F. L. Adding families to the homework equation:a longitudinal study of mathematics achievement. Education and Urban Society, Beverly Hills, v. 43, n. 3, p. 313-338, May 2011. https://doi.org/10.1177/0013124510380236

WEISLEDER, A. et al. Reading aloud and child development: a clusterrandomized trial in Brazil. Pediatrics, Itasca, v. 141, n. 1, e20170723, Jan. 2018. https://doi.org/10.1542/peds.2017-0723 
WILICHOWSKI, T. et al. Building back better: accelerating learning when schools reopen, and what Kenya's Tusome program can teach us. Washington, DC: World Bank Group, 2020. Disponível em: https://blogs.worldbank.org/ education/building-back-better-accelerating-learning-when-schools-reopenand-what-kenyas-tusome?CID=WBW_AL_BlogNotification_EN_EXT. Acesso em: 15 maio 2020.

WILMORE, C.; SLATE, J. Texas elementary school academic achievement as a function of school calendar type. Journal of Educational Policy, Planning, and Administration, [s. 1.], v. 2, n. 1, p. 24-38, 2012.

WU, D. Disentangling the effects of the school year from the school day: evidence from the TIMSS Assessments. Education Finance and Policy, Cambridge, v. 15, n. 1, p. 104-135, 2020. https://doi.org/10.1162/ edfp_a_00265

YEŞIL DAĞLI, Ü. Effect of increased instructional time on student achievement. Educational Review, [s. 1.], v. 71, n. 4, p. 501-517, Apr. 2018. https://doi.org/10.1080/00131911.2018.1441808

ZUCKERMAN, B.; AUGUSTYN, M. Books and reading: evidence-based standard of care whose time has come. Academic Pediatrics, New York, v. 11, n. 1, p. 11-17, 2011. https://doi.org/10.1016/j.acap.2010.09.007.

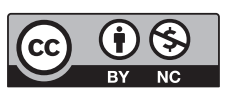

\section{Informações dos autores}

João Batista Araujo e Oliveira: Psicólogo. Doutor em Educação e Presidente do Instituto Alfa e Beto. Contato: jmeduc@gmail.com

iD https://orcid.org/0000-0003-2019-7333

Matheus Gomes: Mestre em Economia Aplicada pela Universidade Federal de Ouro Preto. Pesquisador na área de Educação no IDados. Contato: matheus.souza@idados.id

iD https://orcid.org/0000-0001-5324-4760

Thais Barcellos: Mestre em Economia pela Universidade de São Paulo. Pesquisadora do IDados. Contato: thais@idados.id

iD https://orcid.org/0000-0002-7827-7400 